\title{
Neurological variability in chemotherapy-induced posterior reversible encephalopathy syndrome associated with thrombotic microangiopathy: Case reports and literature review
}

\author{
CHEN MAKRANZ ${ }^{1}$, SALOME KHUTSURAULI ${ }^{2}$, YOSEF KALISH ${ }^{3}$, RUTH ELIAHOU ${ }^{4}$, \\ LUNA KADOURI $^{2}$, JOHN MOSHE GOMORI ${ }^{4}$ and ALEXANDER LOSSOS ${ }^{1}$ \\ ${ }^{1}$ Department of Oncology and Neurology, Leslie and Michael Gaffin Center for Neuro-Oncology, \\ Hadassah-Hebrew University Medical Center; Departments of ${ }^{2}$ Oncology, ${ }^{3}$ Hematology and ${ }^{4}$ Radiology, \\ Sharet Institute for Oncology, Hadassah-Hebrew University Medical Center, Jerusalem 91120, Israel
}

Received June 28, 2017; Accepted September 8, 2017

DOI: $10.3892 / \mathrm{mco} .2017 .1476$

\begin{abstract}
Posterior reversible encephalopathy syndrome (PRES) is a clinical syndrome characterized by headaches, seizures, a confusional state and visual disturbances associated with transient predominantly bilateral posterior white mater magnetic resonance imaging lesions. It is primarily reported in the setting of hypertension, acute renal failure, peripartum eclampsia, autoimmune disease, immunosuppression and chemotherapy. Thrombotic microangiopathy (TMA), including hemolytic uremic syndrome (HUS) and thrombotic thrombocytopenic purpura (TTP) has also been reported as potential PRES inducer. The present study reviews two cases of patients with PRES, associated with TMA caused by chemotherapy. Their clinical and imaging data, and the relevant literature were reviewed. Patient 1 presented with TMA-induced PRES following mitomycin-C for metastatic colon adenocarcinoma. Treatment with steroids, plasma exchange, intravenous immunoglobulins, aspirin, antihypertensive drugs, and diuretics resulted in resolution of the neurological and imaging deficits. Patient 2 presented with TMA-induced PRES following gemcitabine for metastatic breast carcinoma. Treatment was ineffective and the patient deteriorated despite verapamil, dexamethasone, and plasma exchange. In this report, the relevant literature regarding pathogenesis, treatment and prognosis of chemotherapy-induced PRES associated with TMA was reviewed. We conclude that several chemotherapy agents may cause PRES through various pathogenic mecha-
\end{abstract}

Correspondence to: Dr Chen Makranz, Department of Oncology and Neurology, Leslie and Michael Gaffin Center for Neuro-Oncology, Hadassah-Hebrew University Medical Center, Kiryat Hadassah, POB 12000, Jerusalem 91120, Israel

E-mail: chenmak@hadassah.org.il

Key words: gemcitabine, mitomycin-C, plasma exchange, PRES, TMA nisms, leading to clinical variability and divergent response to therapy.

\section{Introduction}

Posterior reversible encephalopathy syndrome (PRES) is a clinical condition characterized by headaches, seizures, confused state and visual disturbances associated with bilateral, predominantly posterior cerebral white matter lesions on magnetic resonance imaging (MRI). PRES is reported in the setting of hypertension, acute renal failure, peripartum, eclampsia, various autoimmune diseases and multiple therapeutic agents (1-10). Thrombotic microangiopathy (TMA), including hemolytic uremic syndrome (HUS) and thrombotic thrombocytopenic purpura (TTP) (11), is also reported as potential PRES inducer $(12,13)$.

As its name suggests, PRES is usually a reversible condition. Nonetheless, it can cause irreversible injury, even after discontinuation of the offending agent. While chemotherapy-induced PRES is also generally reversible, PRES induced by anti-rejection therapy (tacrolimus, cyclosporine) can lead to permanent neurological sequelae $(10,14)$.

To present the extreme diversity of clinical and imaging findings of chemotherapy-induced PRES related to TMA, we report our experience with two patients who developed this condition, and review the literature.

\section{Case report 1}

A 36-year-old woman presented with subacute headaches, dyspnea, and pedal edema 11 weeks following treatment with mitomycin- $\mathrm{C}$ for metastatic adenocarcinoma of the colon. Her primary tumor had been diagnosed 2 years previously, and she was treated with 5-fluorouacil and irinotecan. After treatment failure, a partial colectomy was performed, followed by 5 -fluorouracil, leucovorin, and cisplatin, and later by a combination of 5-fluorouracil (continuous infusion and bolus), leucovorin, and mitomycin-C. An objective partial response was recorded, and mitomycin- $\mathrm{C}$ was stopped at a cumulative dose of $57 \mathrm{mg} / \mathrm{m}^{2} 11$ weeks prior to admission of the patient. 

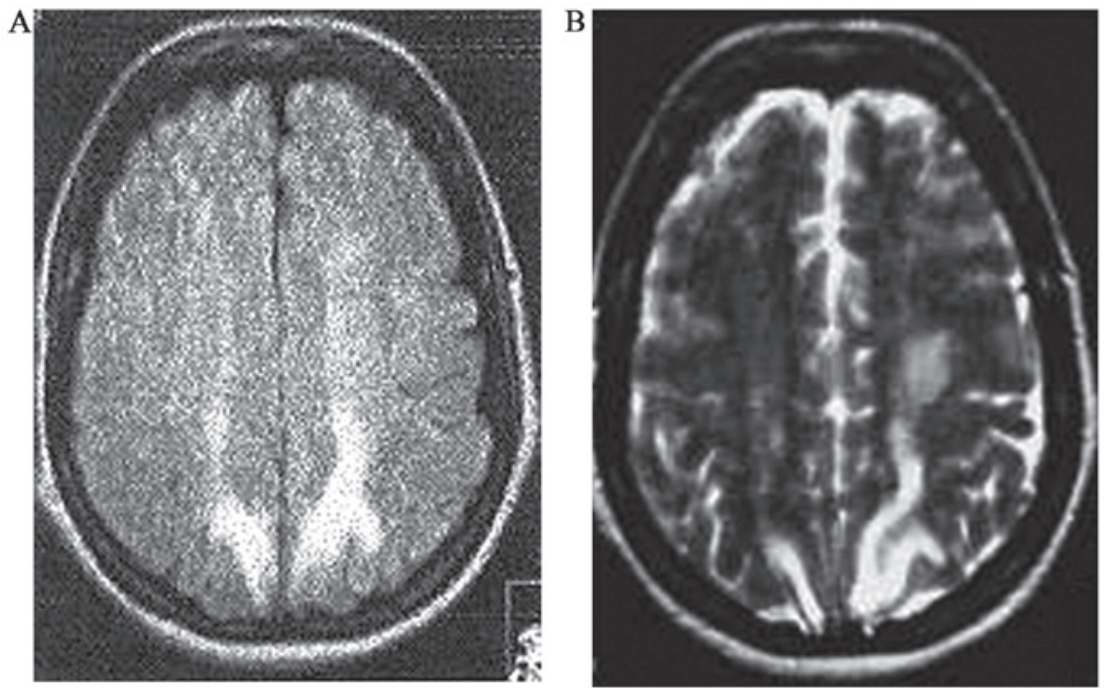

Figure 1. Admission brain MRI of patient 1: Admission brain MRI in a 36-year-old woman with posterior reversible encephalopathy syndrome (PRES) following mitomycin-C (cumulative dose $57 \mathrm{mg} / \mathrm{m}^{2}$. The MRI is 11 weeks after the last dose) for the management of her metastatic colon cancer. The findings are suggestive of posterior reversible encephalopathy syndrome (PRES). (A) Axial T2-weighted fluid-attenuated inversion recovery (FLAIR) and (B) T2-weighted images revealed multiple hypertintense posterior white matter lesions. A scanned version of the images that were prepared many years ago.
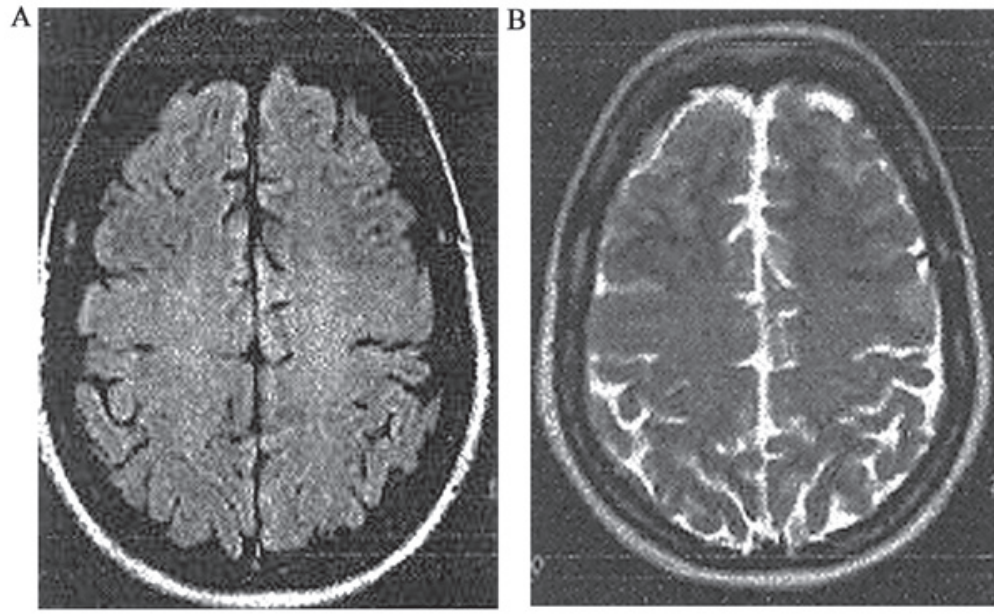

Figure 2. Follow-up brain MRI of patient 1: Brain MRI in the same patient 3 months after diagnosis. (A) Axial FLAIR and (B) T2-weighted images show resolution of the posterior white matter lesions. A scanned version of the images that were prepared many years ago.

Chemotherapy without mitomycin- $\mathrm{C}$ was continued for 6 subsequent weeks.

On examination, 11 weeks from the last dose of mitomycin $\mathrm{C}$, the patient was pale, afebrile, tachypneic, and tachycardic, with signs of bilateral pleural effusion, hepatomegaly, pitting edema, hypertension of 240/120 $\mathrm{mmHg}$, and a grade II hypertensive retinopathy. Blood tests showed hemolytic anemia with $9 \mathrm{~g} / \mathrm{dl} \mathrm{Hg}(12-16 \mathrm{~g} / \mathrm{dl})$, accompanied by lactic acid dehydrogenase $(\mathrm{LDH})$ of $6,400 \mathrm{u} / 1$ (240-480 U/1), total bilirubin of $28 \mu \mathrm{mol} / 1$ (0-17 $\mu \mathrm{mol} / \mathrm{l})$, and thrombocytopenia of 45 10e9/1 (140-40010e9/1), with numerous schistocytes on peripheral blood smear. She also had acute renal failure with creatinine of $309 \mu \mathrm{mol} / 1$ (46-92 $\mu \mathrm{mol} / 1)$ and blood urea nitrogen (BUN) $17 \mathrm{mmol} / 1$ (0-8.3 mmol/l). Activity levels of ADAMTS13 (a disintegrin and metalloproteinase with thrombospondin type 1 motives) activity levels were normal. This combination and the previous maximal mitomycin- $\mathrm{C}$ dose was consistent with the diagnosis of TMA induced by
mitomycin-C $(11,15)$. Despite maximal antihypertensive treatment, the patient's blood pressure remained uncontrolled and she became acutely confused with cortical visual deficit and generalized tonic-clonic seizures that responded to intravenous phenytoin. Brain MRI demonstrated extensive hyperintense posterior white matter lesions on T2 and T2/fluid-attenuated inversion recovery (FLAIR) weighted images typical of PRES (Fig. 1).

Intensive treatment with plasma exchange, steroids, IV immunoglobulin, aspirin, and a combination of antihypertensive agents and diuretics was initiated. Significant clinical and laboratory improvement was observed after 6 weeks. The patient became neurologically intact and repeat brain MRI disclosed resolution of the white matter lesions, with the exception of a small residual left parietal insult (Fig. 2). The patient succumbed 1 year later to her malignant disease. Widespread systemic metastases, with no brain involvement, was the cause of her death. 

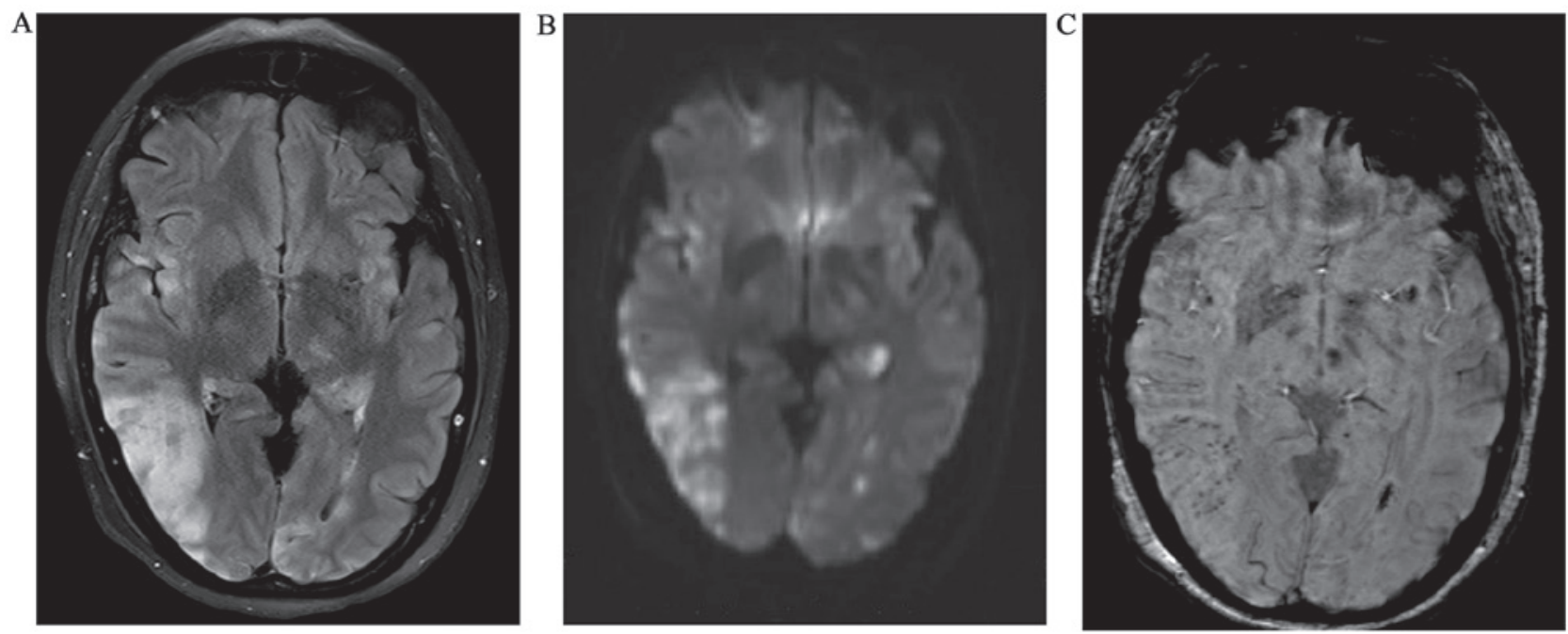

Figure 3. Admission brain MRI of patient 2: Admission brain MRI of a 47-year-old woman with PRES who had undergone treatment with gemcitabine (cumulative dose of $15,000 \mathrm{mg} / \mathrm{m}^{2}$ ) for metastatic breast cancer. (A) FLAIR image depicting multiple foci and diffuse areas of abnormal signal in the subcortical white matter and left median thalamus, as well as swelling of the right occipito-temporal gyri. (B) Diffusion-weighted (DWI) MRI showing multiple posterior white matter lesions with restricted diffusion corresponding to the white matter abnormalities, indicating ischemic change. (C) Susceptibility-weighted images (SWI) show multiple hemorrhagic foci in the brain parenchyma.

\section{Case report 2}

A 47-year-old woman with metastatic breast cancer to the bones and lymph nodes of 7 years duration presented with subacute dysphasia and right hemiparesis, which progressed to full aphasia and hemiplegia over the next 2 weeks. Her neurological deterioration began 1 month after retreatment with gemcitabine (cumulative dose of $15,000 \mathrm{mg} / \mathrm{m}^{2}$ ), which was initially instituted with carboplatin 8 months earlier. Cerebral MRI demonstrated T2/FLAIR hyperintense lesions in the left frontal lobe, the right insular region, and the parieto-occipital region bilaterally. This was accompanied by restricted diffusion and hemorrhagic components, and was compatible with PRES (Fig. 3). Blood pressure was normal. Tests revealed anemia with $\mathrm{Hg}$ of $7.4 \mathrm{~g} / \mathrm{dl}$, a normal platelet count, normal white blood cell (WBC) count, mild bilirubin elevation of $24 \mu \mathrm{mol} / 1$ ( $0-17 \mu \mathrm{mol} / 1)$, and significant elevation of $\mathrm{LDH}$ to $1,608 \mathrm{U} / 1$. Creatinine, BUN, and lactate were normal. Prothrombin time (PT) and partial thromboplastin time (PTT) were normal. Circulating anticoagulants and anticardiolipids were negative and haptoglobin was normal at $137 \mathrm{mg} \%$ (30-200 mg\%).

Treatment with a low dose of verapamil and dexamethasone was initiated. However, despite a transient neurological improvement, the patient deteriorated clinically. Repeat MRI showed worsening of ischemic and hemorrhagic changes. This was accompanied by hemolytic anemia with $\mathrm{Hg}$ of $8 \mathrm{~g} / \mathrm{dl}$, thrombocytopenia of $127 \times 10 \mathrm{e} 9 / 1$, and progressive elevations in values of bilirubin to $50 \mu \mathrm{mol} / 1$ and $\mathrm{LDH}$ to $2,057 \mathrm{U} / 1$. Blood smear revealed a large number of schistocytes, indicating hemolysis secondary to TMA. Plasma activity levels of ADAMTS13 activity levels were normal, compatible with gemcitabine-induced TMA $(11,15)$. Spine MRI ruled out leptomeningeal infiltration. The patient was subsequently treated with 3 courses of plasma exchange and blood products, but her condition worsened and she became unconscious. She died 3 weeks after admission.

\section{Discussion}

We describe 2 patients with chemotherapy-induced TMA who developed PRES that was documented by imaging, and who had very different clinical characteristics. One patient developed reversible PRES associated with TMA 11 weeks after her last mitomycin-C treatment. The other patient was diagnosed with TMA-induced irreversible and fatal PRES following re-administration of gemcitabine.

Mitomycin-C-induced PRES. Mitomycin-C is an antineoplastic antibiotic that has been reported to cause TMA (HUS/TTP-like) in 4-15\% of patients. Most cases occur within 4 months after the last dose. Toxicity is cumulative and dose-dependent, and increases at a dose of $>50 \mathrm{mg} / \mathrm{m}^{2}$ (15-17), as shown in patient 1. Mitomycin-C-induced TMA results from endothelial damage (18), which is at least partly due to formation of platelet-aggregating immune complexes and complement-fixing circulating immune complexes (19). To the best of our knowledge, PRES was not previously reported following treatment with mitomycin-C and may possibly be related to the mitomycin-C-induced TMA. Since the pathogenesis involves both platelet-aggregating and complement-fixing circulating immune complexes, treatment should include plasma exchange, steroids and anti-platelets agents, as in patient 1 .

Gemcitabine-induced PRES. PRES associated with gemcitabine was first documented in 2001 (20) and was reported also after its re-administration (20-22), as in patient 2. Although most instances of PRES due to immunosuppressive agents occur within 2 weeks of treatment initiation or dose increase, it may also occur a few months later (1), as in patient 2 .

According to the literature, PRES associated with gemcitabine is reversible, although in some instances it can result in neurological sequela. Of 12 cases reported in the 
literature, 7 had complete recovery (21,23-26), 4 had partial recovery $(20,27)$, and only one case was fatal due to a hospitalization complication after significant neurological improvement was achieved (7). Gemcitabine-induced PRES may be related to microvascular injury $(7,20,28-30)$ or may develop secondary to TMA. The median cumulative gemcitabine dose associated with TMA is $>20,000 \mathrm{mg} / \mathrm{m}^{2}$ (31). However, prior treatment with chemotherapeutic agents known to cause TMA, such as mitomycin- $\mathrm{C}$ and cisplatin, has been suggested to increase the likelihood of developing TMA after gemcitabine, and to reduce the indicated cumulative dose of the agent (32-35). Indeed, patient 2 had been treated with carboplatin and gemcitabine a few months before her presentation. Gemcitabine induced TMA is believed to result from dysregulation of the complement system, and respond to complement inhibition by eculizumab (36-38). Since no antibody-mediated mechanism has been established, it is unlikely that plasma exchange would be beneficial $(36,39)$, as shown in patient 2 .

Clinical course and prognosis of chemotherapy-induced PRES. Our report documents wide variability in the clinical course of chemotherapy-induced PRES by different agents. Although as its name suggests, PRES is mostly reversible, in some patients the sequelae may be irreversible and even fatal. Patient 1 had full recovery after discontinuation of the offending agent and following plasma exchange with administration of steroids and aspirin. By contrast, patient 2 deteriorated despite discontinuation of the offending agent and treatment with verapamil, steroids, and plasma exchange. This variability is possibly related to the different pathogenic mechanisms attributed to the causative agents, which may also explain differences in the response to therapy. Clinical deterioration with fatal outcome may result from cerebral edema, intracerebral hemorrhage, or as a complication of the underlying condition $(10,14,40)$, such as progressive hemolysis, as in patient 2 .

Prognostic biochemical and imaging markers. Although PRES is usually reversible, up to $15 \%$ of cases are fatal (27). Therefore, it is important to classify patients according to their risk factors. However, only a few prognostic markers have been identified so far, including creatinine and LDH, with their elevation indicating a worse prognosis $(14,41)$. Furthermore, LDH is regarded as an early biochemical marker of PRES, predicting the degree of brain edema in eclampsia and in cancer patients receiving chemotherapy (41). $\mathrm{LDH}$ was elevated in both of our patients, and was not prognostic. This is explained by the fact that both had hemolysis, indicating that LDH is not prognostic in PRES caused by TMA.

In addition to biochemical markers, MRI findings of hemorrhages and restricted diffusion on ADC mapping, suggesting cytotoxic as opposed to vasogenic edema, is also correlated with a worse prognosis (14), as in our patient 2 .

In conclusion, the pathogenic mechanisms in chemotherapy-induced PRES vary, depending on the agent, leading to marked differences in clinical course, prognosis, and outcome.

\section{References}

1. Roth C and Ferbert A: The posterior reversible encephalopathy syndrome: what's certain, what's new? Pract Neurol 11: 136-144, 2011.
2. Hinchey J, Chaves C, Appignani B, Breen J, Pao L, Wang A, Pessin MS, Lamy C, Mas JL and Caplan LR: A reversible posterior leukoencephalopathy syndrome. N Engl J Med 334: 494-500, 1996.

3. Strandgaard S and Paulson OB: Cerebral autoregulation. Stroke 15: 413-416, 1984.

4. Ozcan C, Wong SJ and Hari P: Reversible posterior leukoencephalopathy syndrome and bevacizumab: N Engl J Med 354: 980-982; discussion 980-982, 2006.

5. Allen JA, Adlakha A, and Bergethon PR: Reversible posterior leukoencephalopathy syndrome after bevacizumab/FOLFIRI regimen for metastatic colon cancer. Arch Neurol 63: 1475-1478, 2006.

6. Bodkin CL and Eidelman BH: Sirolimus-induced posterior reversible encephalopathy. Neurology 68: 2039-2040, 2007.

7. Rajasekhar A and George TJ: Gemcitabine-induced reversible posterior leukoencephalopathy syndrome: a case report and review of the literature. Oncologist 12: 1332-1335, 2007.

8. Junna MR and Rabinstein AA: Tacrolimus induced leukoencephalopathy presenting with status epilepticus and prolonged coma. J Neurol Neurosurg Psychiatry 78: 1410-1411, 2007.

9. Kozak OS, Wijdicks EF, Manno EM, Miley JT and Rabinstein AA: Status epilepticus as initial manifestation of posterior reversible encephalopathy syndrome. Neurology 69: 894-897, 2007.

10. Stott VL, Hurrell MA and Anderson TJ: Reversible posterior leukoencephalopathy syndrome: a misnomer reviewed. Intern Med J 35: 83-90, 2005.

11. George JN and Nester CM: Syndromes of thrombotic microangiopathy. New England Journal of Medicine 371: 654-666 2014.

12. Kwon S, Koo J and Lee S: Clinical spectrum of reversible posterior leukoencephalopathy syndrome. Pediatric Neurology 24: 361-364, 2001.

13. Bhat RA, Wani Z, Baasit S and Khan I: Clinical course, laboratory parameters and outcome of TTP pediatric patients presenting with posterior reversible encephalopathy syndrome: Renal failure 37: 974-979, 2015.

14. Covarrubias DJ, Luetmer PH and Campeau NG: Posterior reversible encephalopathy syndrome: prognostic utility of quantitative diffusion-weighted MR images: Am J Neuroradiol 23: 1038-1048, 2002.

15. Al-Nouri ZL, Reese JA, Terrell DR, Vesely SK and George JN: Drug-induced thrombotic microangiopathy: a systematic review of published reports: Blood 125: 616-618, 2015.

16. Valavaara $\mathrm{R}$ and Nordman E: Renal complications of mitomycin- $\mathrm{C}$ therapy with special reference to the total dose: Cancer 55: 47-50, 1985.

17. Reese JA, Bougie DW, Curtis BR, Terrell DR, Vesely SK, Aster RH and George JN: Drug-induced thrombotic microangiopathy: Experience of the Oklahoma registry and the BloodCenter of Wisconsin. American Journal of Hematology 90: 406-410, 2015

18. Groff JA, Kozak M, Boehmer JP, Demko TM and Diamond JR: Endotheliopathy: a continuum of hemolytic uremic syndrome due to mitomycin therapy. Am J Kidney Dis 29: 280-284, 1997.

19. Mistry B, Kimmel PL, Hetzel PC, Phillips TM and Braden GL: The role of circulating immune complexes and biocompatibility of staphylococcal protein A immunoadsorption in mitomycin-C-induced hemolytic uremic syndrome: Am J Kidney Dis 44: e50-58, 2004.

20. Russell MT, Nassif AS, Cacayorin ED, Awwad E, Perman W and Dunphy F: Gemcitabine-associated posterior reversible encephalopathy syndrome: MR imaging and MR spectroscopy findings. Magn Reson Imaging 19: 129-132, 2001.

21. Larsen FO and Hansen SW: Severe neurotoxicity caused by gemcitabine treatment. Acta Oncol 43: 590-591, 2004.

22. Govindarajan R, Adusumilli J, Baxter DL, El-Khoueiry A and Harik SI: Reversible posterior leukoencephalopathy syndrome induced by RAF kinase inhibitor BAY 43-9006: J Clin Oncol 24: e48-e 48, 2006.

23. How J, Blattner M, Fowler S, Wang-Gillam A and Schindler SE: Chemotherapy-associated posterior reversible encephalopathy syndrome: a case report and review of the literature. The neurologist 21: 112-117, 2016.

24. Cherniawsky H, Merchant N, Sawyer M and Ho M: A case report of posterior reversible encephalopathy syndrome in a patient receiving gemcitabine and cisplatin. Medicine 96: e5850, 2017.

25. Bhatt A, Farooq MU, Majid A and Kassab M: Chemotherapy-related posterior reversible leukoencephalopathy syndrome. Nat Rev Neurol 5: 163, 2009. 
26. Truong QV, Abraham J, Nagaiah G, Newton M and Veltri L: Gemcitabine associated with posterior reversible encephalopathy syndrome (PRES): a case report and review of the literature. Clin Adv Hematol Oncol 10: 611-613, 2012.

27. Kabre RS and Kamble KM: Gemcitabine and Cisplatin induced posterior reversible encephalopathy syndrome: A case report with review of literature. J Res Pharm Pract 5: 297-300, 2016.

28. Baker WJ, Royer GL, Jr and Weiss RB: Cytarabine and neurologic toxicity. J Clin Oncol 9: 679-693, 1991.

29. Ito Y, Arahata Y, Goto Y, Hirayama M, Nagamutsu M, Yasuda T, Yanagi T and Sobue G: Cisplatin neurotoxicity presenting as reversible posterior leukoencephalopathy syndrome. Am J Neuroradiol 19: 415-417, 1998.

30. Beck WT and Kuttesch JF: Neurological symptoms associated with cyclosporin plus doxorubicin. Lancet 340: 496, 1992.

31. Walter RB,JoergerM, and Pestalozzi BC: Gemcitabine-associated hemolytic-uremic syndrome. Am J Kidney Dis 40: e16.1-e16.6, 2002.

32. Bharthuar A, Egloff L, Becker J, George M, Lohr JW, Deeb G and Iyer RV: Rituximab-based therapy for gemcitabine-induced hemolytic uremic syndrome in a patient with metastatic pancreatic adenocarcinoma: a case report. Cancer Chemother Pharmacol 64: 177-181, 2009.

33. Muller S, Schutt P, Bojko P, Nowrousian MR, Hense J, Seeber S and Moritz T: Hemolytic uremic syndrome following prolonged gemcitabine therapy: report of four cases from a single institution. Ann Hematol 84: 110-114, 2005.

34. Walter RB, Joerger M and Pestalozzi BC: Gemcitabine-associated hemolytic-uremic syndrome. Am J Kidney Dis 40: e16, 2002.

35. Fung MC, Storniolo AM, Nguyen B, Arning M, Brookfield W and Vigil J: A review of hemolytic uremic syndrome in patients treated with gemcitabine therapy. Cancer 85: 2023-2032, 1999.

36. Starck M and Wendtner CM: Use of eculizumab in refractory gemcitabine-induced thrombotic microangiopathy. Br J Haematol 164, 894-896, 2013.
37. Turner JL, Reardon J, Bekaii-Saab T, Cataland SR and Arango MJ: Gemcitabine-associated thrombotic microangiopathy: response to complement inhibition and reinitiation of gemcitabine. Clin Colorectal Cancer 16: e119-e122, 2016.

38. Rogier T, Gerfaud-Valentin M, Pouteil-Noble C, Taleb A, Guillet M, Noel A, Broussolle C and Seve P: Clinical efficacy of eculizumab as treatment of gemcitabine-induced thrombotic microangiopathy: A case report. Rev Med Interne 37: 701-704, 2016.

39. Gore EM, Jones BS and Marques MB: Is therapeutic plasma exchange indicated for patients with gemcitabine-induced hemolytic uremic syndrome? J Clin Apher 24: 209-214, 2009.

40. Ay H, Buonanno FS, Schaefer PW, Le DA, Wang B, Gonzalez G and Koroshetz WJ: Posterior leukoencephalopathy without severe hypertension: utility of diffusion-weighted MRI. Neurology 51: 1369-1376, 1998.

41. Lv C and Gao B: Serum lactate dehydrogenase as a predictor of outcome in posterior reversible encephalopathy syndrome: imperative to unify. Am J Neuroradiol 36: e29-e30, 2015. 